\title{
The Energy Storage Properties of Supercapacitors with Carbon-Based Electrodes
}

\author{
A.P. Durajski ${ }^{a, *}$, K. Gruszka ${ }^{a}$, K. GizA $^{b}$ And P. Niegodajew ${ }^{c}$ \\ ${ }^{a}$ Częstochowa University of Technology, Department of Physics, \\ al. Armii Krajowej 19, 42-201 Częstochowa, Poland \\ ${ }^{b}$ Częstochowa University of Technology, Department of Materials Engineering, \\ al. Armii Krajowej 19, 42-201 Częstochowa, Poland \\ ${ }^{c}$ Częstochowa University of Technology, Department of Thermal Machinery, \\ al. Armii Krajowej 19, 42-201 Częstochowa, Poland
}

Doi: 10.12693/APhysPolA.138.148

*e-mail: adurajski@wip.pcz.pl

\begin{abstract}
Activated carbon has been found to be a promising material for energy storage systems, especially for supercapacitors which overcome the performance of the conventional capacitors as they can store tremendous amounts of energy. Unlike typical batteries, which use chemical reactions to accumulate an electric charge, supercapacitors with activated carbon electrodes are based on electrostatics. Herein, we report properties of double-layer supercapacitor with highly porous activated carbon felt electrodes capable of achieving not only high power density but also a high energy density. In particular, we investigated the influence of the modification of electrodes with graphite flakes on the electrical capacity of supercapacitors. Due to attractive experimental results, this work is an important step towards large scale commercialization of carbon-based supercapacitor development technology.
\end{abstract}

topics: supercapacitors, energy storage, carbon-based electrodes

\section{Introduction}

Facing shortages in petroleum reserves, worries about the effects of greenhouse gas emissions and a rapidly growing energy demand, it is observed that a considerable amount of effort is put into the research, development, and deployment of continuous progress in high-performance energy storage systems aimed at minimizing the energy waste. Particular attention is paid to the development of such electrochemical storage devices as rechargeable batteries and supercapacitors (SCs). The latter ones are capable of storing a considerably greater amount of energy than conventional capacitors, however, still for a short time [1]. Currently, most of the studies are focussed on developing new anode materials being capable to improve the overall performance of SCs [2]. Even though a multitude of materials have been examined it seems difficult to indicate a material being able to replace commercially applied activated carbons (ACs) - as an anode material for SCs - owing to its high surface area, high stability and good thermal, and electric conductivity $[3,4]$. AC SCs are characterized by a high surface area and offer high both electrical conductivity and voltage stability, temperature stability, long cycling stability and most importantly at low cost [5]. Worthy mentioning is that AC SCs can be produced using biomass raw material which can be beneficial twofold: first, due to environmental aspects; second, biomass raw material is inexpensive and widely available so using it will additionally lower the production costs of SCs [6,7].

There are several methods allowing attaining a layered porous structure and among others, chemical activation seems to be the most reasonable choice due to its simplicity and low costs $[8,9]$. As revealed from the most recent review papers $[10,11]$, most of the research works are focused on searching for new anode materials or focus on developing the methods allowing to enhance the overall performance of SCs as for instance by modifying existing materials.

In the present work, we focussed on improving double-layer SCs performances by modifying AC with graphite flakes. Our preliminary results showed that introduced AC modification is a promising solution that can be easily applied to enhance the overall performance of electrode materials for electrochemical SCs in order to ensure a greater potential for practical applications in modern electronic devices.

\section{Preparation of supercapacitors}

Four identical sets of two cylindrical shape electrodes with dimensions of $2 \mathrm{~mm}$ in height, $5.5 \mathrm{~mm}$ in diameter, and $3.0-3.5 \mathrm{mg}$ in weight were prepared from a highly porous activated carbon felt made of a tangle of carbon fibers. The elemental 


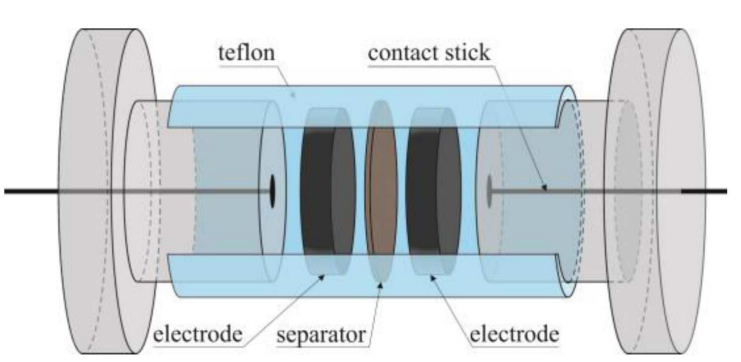

Fig. 1. Schematic design of the Swagelok test cell.

analysis showed that the single fiber, beyond carbon (weight percentages of $88.78 \%$ ), consists of oxygen $(9.06 \%)$, phosphorus $(1.02 \%)$, sodium $(0.73 \%)$, and sulfur $(0.41 \%)$ atoms. The first set of electrodes was cleaned under ultrasonication for $30 \mathrm{~min}$ in $4 \mathrm{ml}$ of pure ethanol to remove attached dust particles. Afterward, AC felts were dried at $60^{\circ} \mathrm{C}$ for $6 \mathrm{~h}$ (after such a time a further drying leads to no change in mass). The second, third and fourth set of electrodes were prepared through modification of $\mathrm{AC}$ felt electrodes adopting the same procedure as stated above but with graphite flakes added into the ethanol. The concentrations of graphite flakes were maintained at $0.125,0.25$, and $0.5 \mathrm{mg} / \mathrm{ml}$, for second, third and fourth sample, respectively. After ultrasonication for $30 \mathrm{~min}$, modified activated carbon samples were also dried and then weighed to get their actual mass after modification. Finally, the weights of the investigated electrodes were 2.50 , $2.50,3.67$, and $4.00 \mathrm{mg}$, respectively.

In the next step, a trilayer polypropylenepolyethylene-polypropylene separator with a diameter slightly greater than that of the one of the electrodes was cut out to avoid direct electrodeelectrode contact. The two electrodes and separator were soaked into the $6 \mathrm{M} \mathrm{KOH}$ aqueous electrolyte for $2 \mathrm{~h}$ and then taken out from the electrolyte solution and assembled into a SC in two-electrodes Swagelok test cell as presented in Fig. 1. The fabricated symmetrical SCs were denoted as S1, S2, S3, and $\mathrm{S} 4$, respectively.

\section{Experimental results}

The microstructure of the prepared electrodes was investigated by scanning electron microscopy (SEM) using an acceleration voltage of $20 \mathrm{kV}$. Figure $2 \mathrm{a}$ presents a global view of pristine $\mathrm{AC}$ felt which is similar in each case. Figure $2 \mathrm{~b}$ shows a single fiber of AC felt which as can be seen is made of about $3 \mu \mathrm{m}$ threads. Figure 2c and d presents material before and after modification, respectively. From a higher magnification SEM image, one can see that the carbon fiber surface of the pristine electrode (Fig. 2c) material is then decorated by graphite flakes (Fig. 2d) in the modification process. The mean diameter of the attached graphite flakes is about $1 \mu \mathrm{m}$.
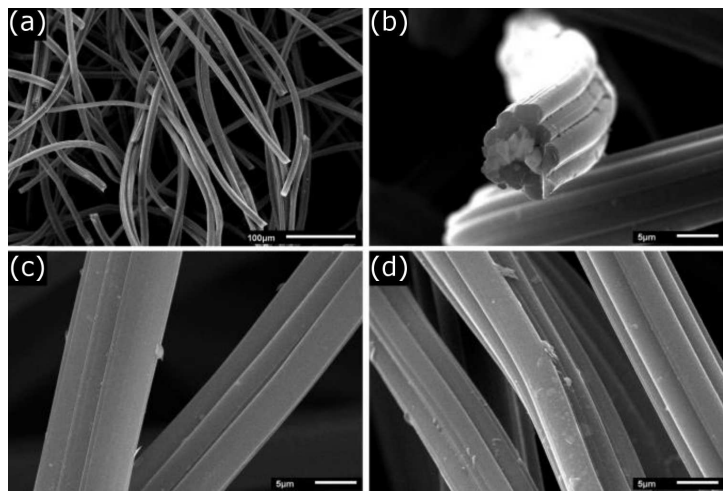

Fig. 2. SEM images obtained for the samples S1 (a, c) and S3 (b, d). Magnifications are 250× (a) and $3000 \times(b, c, d)$.

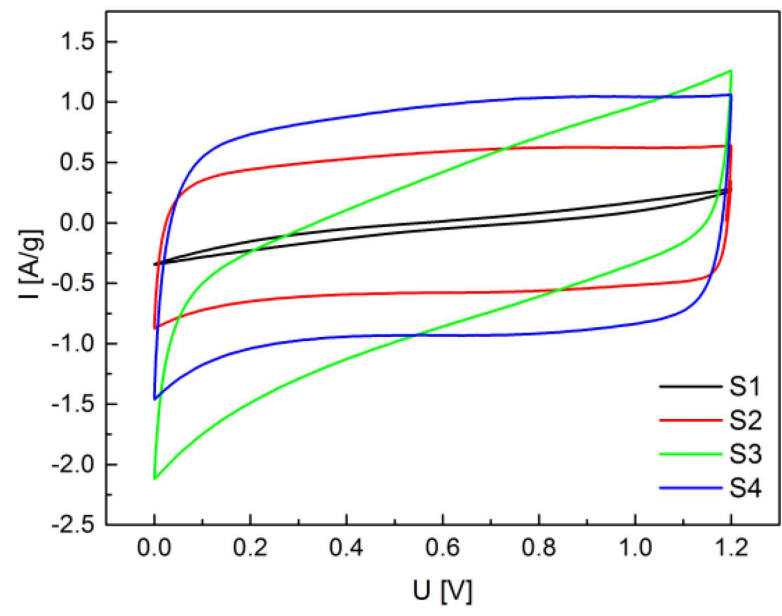

Fig. 3. Cyclic voltammetry for all investigated SCs with $6 \mathrm{M} \mathrm{KOH}$ aqueous electrolyte at scan rate of $10 \mathrm{mV} / \mathrm{s}$ and the working voltage window of $1.2 \mathrm{~V}$.

The influence of modifications on the performance of activated carbon electrode-based doublelayer capacitors was studied by cyclic voltammetry $(\mathrm{CV})$ and galvanostatic charge-discharge (GCD) in the two-electrode system. All measurements were carried out at room temperature by a $\mathrm{CH}$ Instruments potentiostat/galvanostat. CV curves of the investigated SCs with a scan rate of $10 \mathrm{mV} / \mathrm{s}$ in the potential window of -0.8 to $+0.4 \mathrm{~V}$ are presented in Fig. 3. The curves of SCs S2, S3, and S4 constructed from modified electrodes are close to quasirectangular shape without any obvious redox peaks, which indicates good capacitive behavior [12]. The $\mathrm{CV}$ plot of the $\mathrm{S} 1$ is very narrow, which is associated with less capacitance than the other samples.

Furthermore, the GCD curves of SCs under a current density of $0.5 \mathrm{~A} / \mathrm{g}$, presented in Fig. 4, have triangle forms with small internal resistance drop, defined as the electrical potential difference between the two ends of a conducting phase during charging/discharging. 


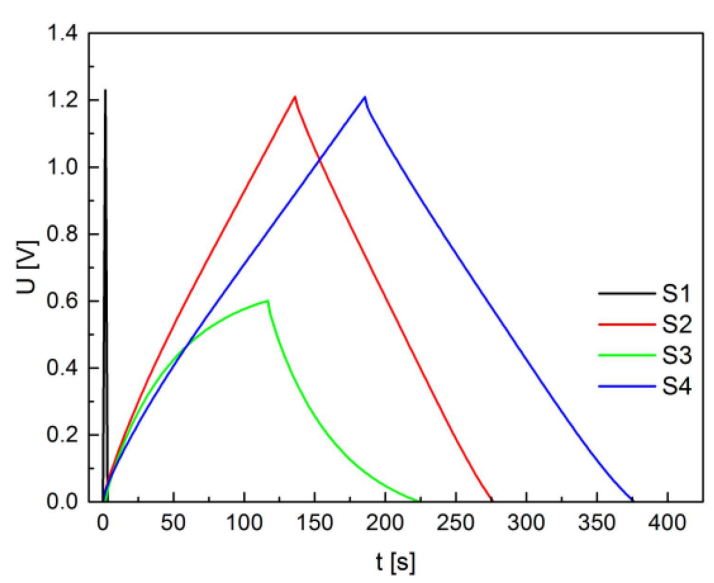

Fig. 4. Galvanostatic charge/discharge curves of SCs S1, S2, S3, and S4 with $6 \mathrm{M} \mathrm{KOH}$ aqueous electrolyte at constant current density of $0.5 \mathrm{~A} / \mathrm{g}$.

The specific capacitance $(C)$ could be calculated from GCD as follows [13, 14]:

$$
C=\frac{4 I \Delta t}{m \Delta V}
$$

where $I$ is the discharge current, $\Delta t$ - the discharging time, $\Delta V$ the operating voltage window, and $m$ the total weight of active materials of two electrodes in the Swagelok cell. The specific capacitance of the investigated SCs at a current density of $0.5 \mathrm{~A} / \mathrm{g}$ can be as high as 116.0, 178.7 and $158.7 \mathrm{~F} / \mathrm{g}$ for S2, S3, and $\mathrm{S} 4$, respectively, which is on average a more than one-hundred-fold higher than the capacitance of SC S1 $(1.33 \mathrm{~F} / \mathrm{g})$ fabricated from the unmodified AC.

Beyond the capacitance, the energy density $E$ and power density $P$ are vital factors for SCs, especially regarding their practical application. These quantities were derived from GCD according to the equations $[15,16]$ :

$$
E=\frac{1}{8} C \Delta V^{2}
$$

and

$$
P=\frac{E}{\Delta t}
$$

Our calculations showed that the energy density of the investigated S1, S2, S3 and S4 SCs reached 0.24, $20.88,8.04,28.56 \mathrm{~W} \mathrm{~h} / \mathrm{kg}$, respectively, whereas the power density at a current density of $0.5 \mathrm{~A} / \mathrm{g}$ was $270 \mathrm{~W} / \mathrm{kg}$ for $\mathrm{S} 3$ and $540 \mathrm{~W} / \mathrm{kg}$ for the remaining SCs. The calculated specific capacitance, energy density and power density of these materials seem to be far better than the previously reported SCs based on graphene-activated carbon [17-19]. This indicates the potential of such modified electrodes for application in electrochemical SCs.

\section{Conclusions}

Activated carbon felt electrodes modified with graphite flakes were tested for the development of material for efficient SCs. As microstructure study showed, the ultrasonication process allowed for flakes to be incorporated on the fiber surface. We found that all the modified SCs had much higher capacitance than the starting material which is connected with the increase of the electrode surface area. A high voltage window of $1.2 \mathrm{~V}$ observed for symmetric AC electrodes cell provides a capacitance of $116.0,178.7$ and $158.7 \mathrm{~F} / \mathrm{g}$, and high energy density of $20.88,8.04,28.56 \mathrm{~W} \mathrm{~h} / \mathrm{kg}$, for S2, S3, and S4 samples, respectively. Even though there is no evident impact of graphite flakes concentration used (its monotonous change lead to non-monotonous change in SCs performance), an increase in SCs key parameters is huge. Therefore, the obtained results are interesting and show the applicability of modified $\mathrm{AC}$ as a low-cost porous material, which could attract interest from the industry for potential application in energy storage.

\section{Acknowledgments}

Authors acknowledge the financial support from the Częstochowa University of Technology under Grant No. BS/MN-200-301/2019.

\section{References}

[1] Y. Gogotsi, P. Simon, Science 334, 917 (2011).

[2] R. Dubey, V. Guruviah, Ionics 25, 1419 (2019).

[3] K. Nomura, H. Nishihara, N. Kobayashi, T. Asada, T. Kyotani, Energy Environ. Sci. 12, 1542 (2019).

[4] Y. Liu, Z. Hu, K. Xu, X. Zheng, Q. Gao, Acta Phys. Chim. Sin. 24, 1143 (2008).

[5] X. Wang, D. Wu, X. Song, W. Du, X. Zhao, D. Zhang, Molecules 24, 2263 (2019).

[6] X. Su, J. Chen, G. Zheng, J. Yang, X. Guan, P. Liu, X. Zheng, Appl. Surf. Sci. 436, 327 (2018).

[7] F. Ma, RSC Adv. 9, 2474 (2019).

[8] A. Bazan-Wozniak, P. Nowicki, R. Pietrzak, Environ. Res. 161, 456 (2018).

[9] G. Sun, L. Qiu, M. Zhu, K. Kang, X. Guo, Ind. Crop. Prod. 125, 41 (2018).

[10] Z. Li, K. Xu, Y. Pan, Nanotechnol. Rev. 8, 35 (2019).

[11] C. Liu, Y. Liu, T. Yi, C. Hu, Carbon 145 , 529 (2019).

[12] X. Hu, L. Fan, G. Qin, Z. Shen, J. Chen, M. Wang, J. Yang, Q. Chen, J. Power Sources 414, 201 (2019).

[13] T. Chen, L. Dai, J. Mater. Chem. A 2, 10756 (2014). 
[14] H. Gupta, S. Chakrabarti, S. Mothkuri, B. Padya, T.N. Rao, P.K. Jain, Mater. Today Proc. 26, 20 (2019).

[15] A. Sarkar, A.K. Singh, D. Sarkar, G.G. Khan, K. Mandal, ACS Sustain. Chem. Eng. 3, 2254 (2015).

[16] J.R. Rani, R. Thangavel, S. Oh, Y.S. Lee, J.-H. Jang, Nanomaterials 9, 148 (2019).

[17] Y. Chen, X. Zhang, H. Zhang, X. Sun, D. Zhang, Y. Ma, RSC Adv. 2, 7747 (2012).
[18] C. Zheng, X.F. Zhou, H.L. Cao, G.H. Wang, Z.P. Liu, RSC Adv. 5, 10739 (2015).

[19] W. Ma, S. Chen, S. Yang, W. Chen, W. Weng, M. Zhu, ACS Appl. Mater. Interfaces 8, 14622 (2016). 